\title{
Global view on functional foods: European perspectives
}

\author{
M. B. Roberfroid* \\ Université Catholique de Louvain, 7A rue du Rondia, B-1348 Louvain-la-Neuve, Belgium
}

\begin{abstract}
In the context of this presentation, European means the European scientific community, which, over the last decade, has been working on the concepts for functional foods, producing a number of documents including a consensus paper, guidelines and scientific publications. The Functional Food Science in Europe (FUFOSE) project has been quite a unique attempt to establish a strong scientific framework to justify the functional food concept, to discover and develop new functional foods that are primarily function-driven, and to substantiate claims scientifically. Being clearly positioned as part of nutrition, the functional food concept is, however, quite distinct from other approaches like food supplementation or food fortification, and functional foods are different from nutraceuticals, pharmafoods, vitafoods and 'alicaments', all terms that are not defined conceptually. Functional foods are food products to be taken as part of the usual diet in order to have beneficial effects that go beyond what are known as traditional nutritional effects. Moreover, these beneficial effects have to be demonstrated scientifically to justify two specific types of claim: the enhanced function claim or the reduction of disease risk claim.

Functional food is a key concept for the future of nutrition as a science because it results from the implementation in nutrition of all the basic scientific knowledge that has accumulated over the past two or three decades. To the benefit of public health this progress cannot be ignored, it needs to be recognized fully and used. But, today, functional food is still mainly a scientific concept that serves to stimulate research and the development of new products.
\end{abstract}

Functional foods: Markers: Claims: FUFOSE

\section{Nutrition: a science for the twenty-first century}

At the beginning of the twenty-first century, the society of abundance, which characterizes most of the occidental/ industrialized world, faces new challenges from increased costs of health care, increased life expectancy, new scientific knowledge and the development of new technologies leading to major changes in life-styles (Table 1). Nutrition, as a science of the twenty-first century, will, in addition to maintaining the emphasis on a balanced diet, target optimum (optimized) nutrition (Milner, 2000), with the objective of maximizing physiological functions in order to ensure both maximum well-being and health and simultaneously minimize the risk of disease throughout the life-span. At the same time, and because of a better understanding of the interactions between genes and nutrition (Kok, 1999), it will have to match the individual's unique biochemical needs with a tailored selection of nutrient intakes for that individual. These interactions include polymorphism and inter-individual variations in response to diet, dietary alteration and modulation of gene expression, and dietary effects on disease risk.

Thus, at the turn of the new century, the major challenge of the science of nutrition is thus to progress from improving life expectancy to improving life quality/wellness.

On the road to optimum (optimized) nutrition, which is an ambitious and long-term objective, 'functional food' is, amongst others, a new, interesting and stimulating concept. It is supported by sound and consensual scientific data generated by the recently developed 'functional food science', which aims to improve dietary guidelines by integrating new knowledge on the interactions between food components and body functions and/or pathological processes.

\section{Functional food: defining the concept}

Functional food cannot be a single well-defined/wellcharacterized entity. Indeed, a wide variety of food products are, or will be in the future, characterized as functional foods, with a variety of components - both classified and not classified as nutrients - affecting a variety of body functions relevant to either a state of well-being and health and/or to the reduction of the risk of a disease. Thus no simple, universally accepted definition of functional food exists or will (ever) exist. Functional food has 
Table 1. List of some challenges to Western societies at the turn of the new century

Costs of health care

Increase in life expectancy costs

Consumer awareness of the importance of nutrition in health and well-being

Technical advances in food processing

Improved knowledge of diet-related diseases

Application of new scientific knowledge in nutrition

thus to be understood as a concept (Roberfroid, 2000). Moreover, if it is function-driven rather than productdriven, the concept is likely to be more universal and not too much influenced by local characteristics or cultural traditions (Diplock et al. 1999).

In scientific as well as in more marketing-oriented literature, functional food has as many definitions as the number of authors referring to it. These definitions go from simple statements, such as:

1. foods that may provide health benefits beyond basic nutrition (IFIC Foundation, 1995) and

2. foods or food products marketed with the message of the benefit to health (Riemersma, 1996),

to very elaborate definitions such as:

1. food and drink products derived from naturally occurring substances consumed as part of the daily diet and possessing particular physiological benefits when ingested (Hillian, 1995);

2. food derived from naturally occurring substances, which can and should be consumed as part of the daily diet and which serves to regulate or otherwise affect a particular body process when ingested (Smith et al. 1996);

3. food similar in appearance to conventional food, which is consumed as part of the usual diet and has demonstrated physiological benefit and/or reduces the risk of chronic disease beyond basic nutritional functions (Health Canada, 1997); and

4. food that encompasses potentially helpful products, including any modified food or food ingredient that may provide a health benefit beyond that of the traditional nutrient it contains (Food and Nutrition Board, 1994).

But whatever definition is chosen, 'functional food' appears as a quite unique concept that deserves a category of its own, a category different from nutraceutical, $\mathrm{f}$ (ph)armafood, medifood, designer food or vitafood, and a category that does not include dietary supplements. It is also a concept that belongs to nutrition and not to pharmacology. Functional foods are and must be foods, not drugs. Moreover, their role regarding disease will, in most cases, be in 'reducing the risk' rather than 'preventing' it (Roberfroid, 2000).

\section{Functional food: a European consensus}

The unique features of a 'functional food' are (Bellisle et al. 1998; Knorr, 1998):

1. a conventional or everyday food,
2. consumed as part of the normal/usual diet,

3. composed of naturally occurring (as opposed to synthetic) components, perhaps in unnatural concentrations or present in foods that would not normally supply them, and

4. having a positive effect on target function(s) beyond nutritive value/basic nutrition,

5. that may enhance well-being and health and/or reduce the risk of disease or provide health benefit so as to improve the quality of life including physical, psychological and behavioural performances, and

6. have authorized and scientifically based claims.

It is in this general context that the European Commission's Concerted Action on Functional Food Science in Europe (FUFOSE), which actively involved a large number of the most prominent European experts in nutrition and related sciences, has been coordinated by the International Life Sciences Institute (ILSI) Europe. It developed in early 1996 to reach a European Consensus on 'Scientific Concepts of Functional Foods', which was published in 1999 (Diplock et al. 1999).

As already indicated above, and because functional food is a concept rather than a well-defined group of food products, that consensus document proposes 'a working definition':

'A food can be regarded as functional if it is satisfactorily demonstrated to affect beneficially one or more target functions in the body, beyond adequate nutritional effects, in a way that is relevant to either improved stage of health and well-being and/or reduction of risk of disease. A functional food must remain food and it must demonstrate its effects in amounts that can normally be expected to be consumed in the diet: it is not a pill or a capsule, but part of the normal food pattern.'

Diplock et al. (1999)

The main aspects of that working definition are:

1. the food nature of functional food - not a pill, a capsule or any form of dietary supplement,

2. the demonstration of effects to the satisfaction of the scientific community,

3 . the beneficial effects on body functions, beyond adequate nutritional effects, that are relevant to an improved state of health and well-being and/or reduction of risk (not prevention) of disease, and

4. the consumption as part of a normal food pattern.

This definition encompasses all main features of functional foods identified above, and it is aimed at stimulating research and development in the field of nutrition so as to contribute adequately to the scientific knowledge that will be required to define optimum (optimized) nutrition by elaborating new dietary guidelines. But it should be emphasized that a functional food will not necessarily be functional for all members of the population, and that matching individual biochemical needs with selected food component intakes may become a key task as we progress in our understanding of the interactions between genes and diet (Kok, 1999). 
From a practical point of view, a functional food can be:

1. a natural food,

2. a food to which a component has been added,

3. a food from which a component has been removed,

4. a food where the nature of one or more components has been modified,

5. a food in which the bioavailability of one or more components has been modified or

6. any combination of these possibilities.

\section{Strategy for development of functional food: functional food science}

Being foods, functional foods need to be safe according to all criteria defined in current food regulations. But in many cases, new concepts and new procedures will need to be developed and validated to assess functional food risks. In Europe, some, but certainly not all, functional foods will be classified as 'novel food' and, consequently, will require the decision tree assessment regarding safety that is described in the EU Novel Food Regulation (European Commission, 1997).

But that regulation does not concern nutritional properties or physiological effects of these novel foods. It is strictly a safety regulation. The requirement for safety is a prerequisite to any functional food development. Indeed the risk versus benefit concept, that is familiar to pharmacologists developing new drugs, does not apply to functional foods except, maybe, in very specific conditions for disease risk reduction when the scientific evidence is particularly strong.

As described in the European consensus document:

'The design and development of functional foods is a key issue, as well as a scientific challenge, which should rely on basic scientific knowledge relevant to target functions and their possible modulation by food components. Functional foods themselves are not universal and a food-based approach would have to be influenced by local considerations. In contrast, a science-based approach to functional food is universal... The functiondriven approach has the science base as its foundation - in order to gain a broader understanding of the interactions between diet and health. Emphasis is then put on the importance of the effects of food components on well-identified and well-characterized target functions in the body that are relevant to well-being and health issue, rather than, solely, on reduction of disease risk.'

Diplock et al. (1999)

By reference to the new concepts in nutrition outlined above, it is the role of 'functional food science' to stimulate research and development of functional foods (Fig. 1).

Referring to basic knowledge in nutrition and related biological sciences, such a development requires the identification and, at least partly, the understanding of the mechanism(s) by which a potential functional food or functional food component can modulate the target function(s) that is/are recognized or proven to be relevant to the state of well-being and health, and/or the reduction of a disease risk. Epidemiological data demonstrating a

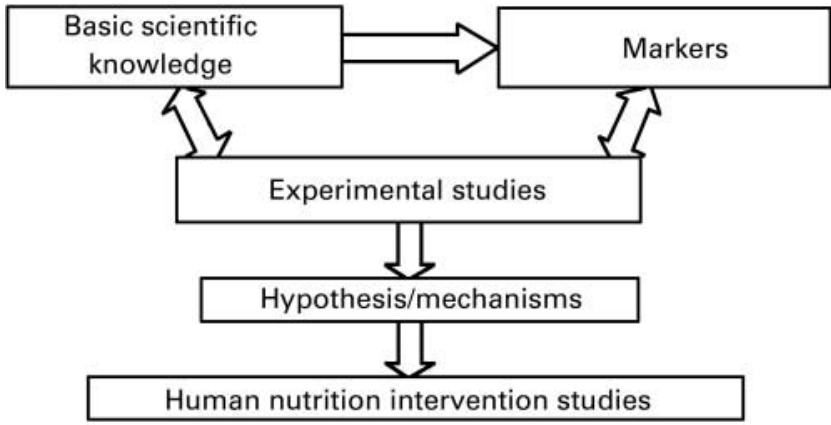

Fig. 1. Schematic representation of the strategy for functional food discovery and development.

statistically validated and biologically relevant relationship between the intake of specific food components and a particular health benefit will, if available, be very useful. The conclusion of the first step will be the demonstration of a functional effect that should serve to formulate hypotheses to be tested in a new generation of human nutrition studies aimed to show that relevant (in terms of dose, frequency, duration, etc.) intake of the specified food will be associated with improvement in one or more target functions, either directly or indirectly in terms of a valid marker of an improved state of wellbeing and health and/or reduced disease risk. If well supported by strong scientific evidence, the conclusion could be a recommendation for improved or new dietary guidelines.

The new-generation human nutrition studies should be hypothesis-driven. But, in many cases, and even though they will have to follow the same basic rules in terms of quality of protocol design, quality of data management and value of statistical analysis, they will differ quite substantially from what is classically referred to as clinical studies. The main differences are that nutrition studies aim at testing the effect of a food as part of the ordinary diet, that they may concern the general population or generally large, at-risk target groups, that they are not diagnostic- or symptoms-based, and that they are not planned to evaluate a risk versus benefit approach. Most of these studies will rely on change(s) in validated/relevant markers to demonstrate a positive modulation of target functions after (long-term) consumption of the potential functional foods. A (double) blind-type of design based on parallel groups, rather than crossing-over, will generally be appropriate. Finally, the long-term consequences of the interaction(s) between functional foods and body function(s) will have to be monitored carefully.

\section{Markers: a key to the development of functional foods}

The development of functional foods will, in most cases, rely on measurements of 'markers'. These markers need to be identified and validated for their predictive value of potential benefits to a target function or the risk of a particular disease. Markers of correlated events are 'indicators', whereas markers representing an event directly involved in the process are 'factors' (Diplock et al. 1999). When related to the risk of a disease, indicators 
and even factors might, in some instances, be equivalent to 'surrogate markers', defined as a biological observation, result or index that predicts the development of a chronic disease (Keystone, 1997). The more is known about the mechanisms leading to health outcomes, the more refined will be the identification of the markers and their appreciation. The markers should be feasible, valid, reproducible, sensitive and specific. They can be biochemical, physiological, behavioural or psychological in nature. But dynamic responses might be as useful as, or more useful than, static or single point measurements. In many cases, a battery of markers might be needed in order to create a decision tree from multiple tests.

These markers, most of which still need to be identified and validated (Fig. 2), should relate to:

1. exposure to the food component under study by measuring the serum, faecal, urine or tissue level of the food component itself or its metabolite(s), or the concentration of an endogenous molecule that is influenced directly by consumption of the food component;

2. target function(s) or biological response, such as the change in serum or other body fluids of the concentration of a metabolite, specific protein, enzyme or hormone, etc. (these first two markers are either indicators or factors);

3. an appropriate endpoint of an improved state of wellbeing and health and/or reduction of a disease risk (such a marker is likely to be a factor rather than an indicator); and

4. individual susceptibility or genetic polymorphism controlling the metabolism and/or the effect of the food component under study (Kok, 1999).

To develop these markers further, a state-of-the-art literature review will be necessary to identify, define and characterize potential markers. Furthermore, the basic scientific knowledge underpinning these markers should be evaluated. The next step will include the assessment of their relevance to physiological functions, well-being and health, and eventually disease risk. A validation will then be necessary both for the methodology and the biological relevance. Finally, the classification as indicator or factor will be made and potential dietary modulations will be demonstrated. New techniques such as those used by molecular and cellular biologists will be useful in identifying target groups who could benefit from the consumption of specific functional foods.

\section{Functional foods and claims: a communication and scientific challenge}

As stated in the European consensus on scientific concepts of functional foods:

'As the relationship between nutrition and health gains public acceptance and as the market for functional foods grows, the question of how to communicate the specific advantages of such foods becomes increasingly important. Communication of health benefits to the public, through intermediates such as health professionals, educators, the media and the food industry, is an essential element in improving public health and in the development of functional foods. Its importance also lies in avoiding problems associated with consumer confusion about health messages. Of all the different forms of communication, those concerning claims made either directly as a statement on the label or package of food products, or indirectly through secondary supporting information - remain an area of extensive discussion.'

Diplock et al. (1999)

It is also the opinion of Hudson (1994) that: 'the links between nutrition science and food product development will flow through to consumers only if the required communication vehicles are put in place'. But the communication of health benefits and other physiological effects of functional foods remains a major challenge because:

1. science should remain the only driving force,

2. messages - claims - must be based on sound, objective and appropriate evidence, and

3. evidence must be consistent, able to meet established scientific standards and plausible.

Moreover, communication in nutrition generally comes from multiple sources that are sometimes contradictory, creating an impression of chaos. And chaotic information often generates ignorance and easily becomes misinformation.

Regarding functional foods, claims associated with specific food products are the preferable means of communicating to consumers. In application of the fundamental

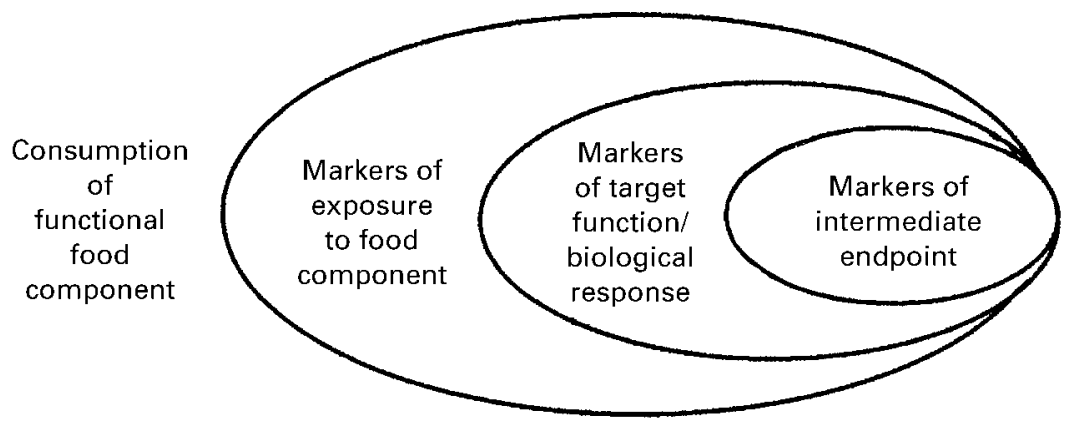

Fig. 2. Schematic representation of the markers that can be used to discover and develop new functional foods. 
principles, any claim must be true and not misleading, it must be scientifically valid, unambiguous and clear to the consumer. These basic principles should be safeguarded without, however, becoming a disincentive to the production of functional foods or to the acceptance of these foods by consumers. Even though a general definition of 'claim' as widely accepted in the field of nutrition is: 'any representation, which states, suggests or implies that a food has certain characteristics relating to its origin, nutritional properties ... or any other quality' (Codex Alimentarius, 1991), one of the difficulties in communicating the benefits of functional foods is that distinct types of claim exist, and that especially the term 'health claim', traditionally used to communicate the benefits of foods, is defined differently in different part of the world. Seeking for clarity, Codex Alimentarius (1991) has classified and defined four different categories of claim, but excluding the term 'health claim', as:

1. relating to dietary guidelines,

2. relating to nutrient content,

3. being comparative (reduced, less, more) and

4. describing nutrient function (contains ..., that contributes to the development of ...).

These claims refer to known nutrients and their role in growth, development and normal function as well as to the concept of adequate nutrition. They are based on established, widely accepted knowledge but they do not refer to a particular effect over and above that expected from consuming a balanced diet. These claims are thus not really helpful to communicate the specific benefits of functional foods. Indeed the claims for functional foods should be based on the scientific classification of markers (indicators and/or factors) for target functions and on the effects on these markers. If such an effect, which goes beyond what could be expected from the established role of diet in growth, development and other normal function in the body, concerns a target function or a biological activity without direct reference to a particular disease or pathological process, claims will be made for 'enhanced function'. But, if the benefit is clearly a reduction of the risk of a disease or pathological process, claims will be made for a 'disease risk reduction'. These two types of claim, which are specific for functional foods, are the 'type A' and 'type B' claims, respectively, as they are described in the European consensus on scientific concepts of functional foods (Diplock et al. 1999). The type A claim is similar to the 'structure-function claim', whereas the type B claim can be regarded as equivalent to the 'health claim' in the USA. Type B claim also corresponds to 'health claim' in Sweden (Swedish Nutrition Foundation, 1996). In its last proposed draft recommendations for the use of health claims, Codex Alimentarius (1999) has included type A and type B claims and defined them as follows.

1. Type A or claims that concern specific beneficial effects of the consumption of foods and their constituents on physiological or psychological functions or biological activities but do not include nutrient function claims. Such claims relate to a positive contribution to health or to a condition linked to health, to the improvement of a function or to modifying or preserving health.

2. Type B or 'risk of disease reduction' claims that concern the reduction of a disease risk related to the consumption of a food or a food constituent in the context of the daily diet that might help reduce the risk of a specific disease or condition.

One of the major issues, still to be resolved especially with these two types of claim, concerns the biological level at which evidence can be accepted as 'satisfactorily demonstrating' an enhanced function or a reduction of disease risk. This evidence should rely on all data available that can be grouped in three categories:

1. biological observations,

2. epidemiological data and

3. intervention studies, mostly based on markers.

But for any given specific food product, supporting evidence for enhanced function or reduction of disease risk might not be available or even not necessary from all three areas (Diplock et al. 1999). All supporting evidence should, however, be:

1. consistent in itself;

2. meet accepted scientific standards of statistical as well as biological significance, especially dose-effect relationship, if relevant;

3. plausible in terms of the relationship between intervention and results, especially in terms of mechanism(s) of action; and

4. provided by a number of sources (including obligatorily human studies) that give consistent findings able to generate scientific consensus.

\section{Food technology and its impact on functional food development}

From the point of view of food processing (Knorr, 1998; Diplock et al. 1999), the development of functional foods will often require an increased level of complexity and monitoring of food processing because

1. new raw materials including those produced by biotechnologies,

2. emerging thermal and non-thermal technologies,

3. new safety issues and

4. integration throughout the entire food chain, especially to ensure preservation and/or enhancement of functionality,

will have to be considered carefully.

The following main areas for technological challenge have been identified.

1. The creation of new food components in traditional and novel raw materials that add or increase functionality. Examples of such challenges are genetic modification, the use of under-utilized or unconventional natural sources (e.g. algae, seaweed) and the development of bioreactors based on immobilized enzymes or live micro-organisms. 
2. The optimization of functional components in raw material and in foods, to ensure maximal preservation of the component(s), modify their function, increase their bioavailability, etc. Examples of such challenges are the development of membrane-processing techniques, the use of controlled and modified atmospheres, the use of high hydrostatic pressure, high-intensity electric field pulse technology and ultrasound treatments.

3. The effective monitoring, throughout the entire food chain, of the amount and functionality of the component(s) in raw materials and foods. Examples of such challenges are the monitoring of microbial viability and productivity for probiotic functions, the development of sensitive markers to record changes in speciation and the interactions with food components during processing, especially fermentation.

\section{Future trends and conclusions}

By reference to the conclusions of the FUFOSE concerted action (Diplock et al. 1999), future trends are as follows.

1. Components in foods have the potential to modulate target functions in the body so as to enhance these functions and/or contribute to reduce the risk of diseases, and 'functional food science' will contribute to human health in the future provided evidence is supported by sound scientific (mostly human) data.

2. Nutritionists and food scientists have the possibility, through the development of functional foods, to offer beneficial opportunities related to well-being and health and reduction of the risk of diseases. But the success of this new approach to nutrition will require the identification, characterization and development of methodologies to measure, and the validation of, relevant markers being indicators or factors to be used in human nutrition studies. The design of such studies still needs to be analysed carefully and developed specifically by reference to, but differently from, classical clinical studies that have been elaborated to help in the development of drugs, not food products.

3. Major target functions in the body that are or can be modulated by specific food products will have to be identified or characterized. The basic science to understand these functions, and how they relate to wellbeing and health or a particular pathological process, needs to be developed so as to give the necessary scientific base to develop new functional food products.

4. Progress in food regulation, which is the means to guarantee the validity of the claims as well as the safety of the food, will have to be made.

On the road to optimized nutrition that will be one of the major challenges of nutrition in the twenty-first century, functional foods have their own role to play. But the development of claims for already existing food products, as well as the development of new products and their own claims, should remain first a scientific challenge and not only a marketing challenge. This is the condition for success to the benefit of both human health and the food industry.

\section{References}

Bellisle F, Diplock AT, Hornstra G, Koletzko B, Roberfroid M, Salminen S \& Saris WHM (editors) (1998) Functional food science in Europe. British Journal of Nutrition 80, Suppl. 1, S1-S193.

Codex Alimentarius (1991) Codex General Guidelines on Claims, CAC/GL 1-1979 Revision 1. Geneva: WHO.

Codex Alimentarius (1999) Proposed Draft Recommendation for the Use of Health Claims. Geneva: WHO.

Diplock AT, Aggett PJ, Ashwell M, Bornet F, Fern EB \& Roberfroid MB (1999) Scientific concepts of functional foods in Europe: consensus document. British Journal of Nutrition 81, Suppl. 1, S1-S27.

European Commission (1997) Novel Food Directive 97/258/CEE.

Food and Nutrition Board, Institute of Medicine, National Academy of Sciences (1994) Opportunities in the Nutrition and Food Sciences [PR Thomas and R Earl, editors]. Washington, DC: National Academy Press.

Health Canada (1997) Policy Options Analysis: Nutraceuticals/ Functional Foods. Ottawa, Ont., Canada: Health Canada, Health Protection Branch, Therapeutic Products Programme and Food Directorate.

Hillian M (1995) Functional foods: current and future market developments. Food Technology International Europe 25-31.

Hudson CB (1994) The food industry's expectation. In Health Claims: Substantiation and Research Needs, pp. 9-11. Melbourne, Australia: ILSI Australasia.

IFIC Foundation (1995) Functional foods: opening the door to better health. Food Insight November/December.

Keystone (1997) The Keystone National Policy Dialogue on Food Nutrition and Health: Executive Summary. Journal of Nutraceuticals, Functional and Medical Foods 1, 11-32.

Knorr D (1998) Functional food science in Europe. Trends in Food Science and Technology 9, 295-340.

Kok FJ (1999) Functional foods: relevance of genetic susceptibility. In Proceedings of Forum on Functional Food, pp. 217-229. Strasbourg: Council of Europe Publishers.

Milner J (2000) Functional foods: the US perspective. American Journal of Clinical Nutrition 71, 1654S-1659S.

Riemersma RA (1996) A fat little earner. Lancet 347, 775-776.

Roberfroid MB (2000) Defining functional foods. In Functional Foods, Concept to Product, pp. 9-28. [GR Gibson and CM Williams, editors]. Cambridge, UK: Woodhead Publishing Ltd.

Smith BL, Marcotte M \& Harman G (1996) A Comparative Analysis of the Regulatory Framework Affecting Functional Food Development and Commercialization in Canada, Japan, the European Union and the United States of America. Ottawa, Ont., Canada: Intersector Alliance Inc.

Swedish Nutrition Foundation (1996) Health Claims in the Labelling and Marketing of Food Products: The Food Industry's Rules (Self-Regulatory Programme). Lund: Swedish Nutrition Foundation. 